\title{
GENERALIZED FREDHOLM OPERATORS AND THE BOUNDARY OF THE MAXIMAL GROUP OF INVERTIBLE OPERATORS
}

\author{
G. W. TREESE AND E. P. KELLY, JR.
}

\begin{abstract}
Let $V$ denote an infinite dimensional Banach space over the complex field and let $G[V]$ denote the subset of bounded operators on $V$ with the property that the null space has a closed complement and the range is closed, where the null space and range are proper subspaces of $V$. Necessary and sufficient conditions for $T \in G[V]$ to be in the boundary, $\mathscr{B}$, of the maximal group, $\mathfrak{R}$, of invertible operators are determined. As a result, $\mathscr{B} \cap G[V]$ is the set of products of operators in $\Re$ and operators in $\mathcal{P}$, where $\mathscr{P}$ is the set of projections other than the identity operator and null operator.
\end{abstract}

1. Introduction. Let $\mathscr{Q}$ denote a Banach algebra, let $\mathscr{N}$ denote the maximal group of invertible elements of $\mathscr{Q}$ and let $\mathscr{B}$ denote the boundary of $\mathscr{N}$. The problem to which this paper is addressed is the determination of necessary and sufficient conditions for an element of $\mathcal{Q}$ to be an element of $\mathcal{B}$. Rhoades [9] considered this problem in the setting in which $\mathscr{Q}$ is the Banach algebra of conservative, infinite, triangular matrices. Sufficient conditions for such matrices to be in $\mathscr{B}$ were given. In [7], Kelly and Hogan considered this problem in the setting in which $\mathbb{Q}$ is the Banach algebra of bounded linear operators defined on an infinite dimensional Banach space $V$ that leave a closed subspace of $V$ invariant. Again, only sufficient conditions for such an operator to be in $\mathscr{B}$ were given. This problem was solved by Feldman and Kadison [5], if $\mathbb{Q}$ is the ring of operators on a Hilbert space. Herein, $\mathbb{Q}$ will denote the Banach algebra $B[V]$, of bounded linear operators defined on an infinite dimensional Banach space $V$ over the complex field.

The terminology generalized Fredholm operator was used by Caradus [4] for those operators in $B[V]$ which have the property that both the null space and the range are closed complemented subspaces of $V$. This set of operators contains the Fredholm operators and, following Caradus, is denoted by $G F[V]$. It is well known [4], [8] that this set of operators is the same as the set of operators in $B[V]$ which have generalized inverses in the sense that $T$ in $B[V]$ is said to have a generalized inverse $S$ in $B[V]$ if and only if $T S T=T$ and $S T S=S$. Also, by way of reference, in a fundamental paper, Atkinson

Received by the editors December 8, 1976 and, in revised form, March 11, 1977.

AMS (MOS) subject classifications (1970). Primary 46L20, 47B99.

Key words and phrases. Banach algebra, maximal group, boundary of maximal group, bounded operator, generalized Fredholm operator, projection, generalized inverse.

(O) American Mathematical Society 1977 
[1] called such operators, i.e., those with generalized inverses, relatively regular. In [1] it was observed that the set of relatively regular operators includes those operators which have inverses in $B[V]$, those operators which have right or left inverses in $B[V]$ and the projection operators. Moreover, in [1] it was shown that generalized nilpotent operators and those completely continuous operators which are not finite-dimensional are not relatively regular.

Now since projections other than the identity and generalized nilpotent operators are both types of operators in $\mathscr{B}$ [7], it is natural to ask what relation exists between $\mathscr{B}$ and $G F[V]$. Denote by $G[V]$ the subset of $B[V]$ consisting of those operators which have the property that the null space has a closed complement and the range is closed, where the null space and range are proper closed subspaces of $V$. Necessary and sufficient conditions for $T$ to be in $\mathscr{B} \cap G[V]$ will be proven, and as a corollary it is shown that $\mathscr{B} \cap G[V]$ is the subset of $B[V]$ consisting of those operators which are products of invertible operators and projections other than the identity operator and null operator.

2. Necessary and sufficient conditions for $T$ to be in $\mathscr{B} \cap G[V]$. For $T \in G[V]$, let $\mathscr{R}(T)$ and $\mathscr{R}(T)$ denote the null space of $T$ and the range of $T$, respectively, and let $V_{1}$ denote a closed complement of $\Re(T)$. Then $T_{1}$, the restriction of $T$ to $V_{1}$, is a linear homeomorphism from $V_{1}$ onto $R(T)$. The main part of the proof of the following theorem is the construction of an extension of $T_{1}$ to an invertible operator in $\Re$. To that end, $\delta$ will denote a set of extensions of $T_{1}$ with certain properties. $\delta$ will be shown to be nonempty and will be partially ordered. Then, a maximal element of $\delta$ will be shown to be the desired extension.

THEOREM. If $T \in G[V]$ and if $V_{1}$ is a closed complement of $\Re(T)$, then the following are equivalent:

(i) there is a sequence $\left\{\tilde{M}_{n}\right\}$ in $\mathfrak{R}$, such that $\left\{\left.\tilde{M}_{n}\right|_{V_{1}}\right\}$ converges to $\left.T\right|_{V_{1}}$ and the sequence $\left\{\left\|\tilde{M}_{n}^{-1}\right\|\right\}$ is bounded;

(ii) there is an invertible $S^{\prime} \in \Re$, such that $\left.S^{\prime}\right|_{V_{1}}=\left.T\right|_{V_{1}}$;

(iii) $T \in \mathscr{B}$;

(iv) $T \in G F[V]$ with $\mathfrak{x}(T)$ linearly homeomorphic to a closed complement of $\mathcal{R}(T)$.

Proof. ((i) implies (ii).) Define a new norm $\|\cdot\|^{\prime}$ on $V=V_{1} \oplus \Re(T)$ by $\|x\|^{\prime}=\left\|x_{1}\right\|+\left\|x_{0}\right\|$, where $x=x_{1}+x_{0}$ with $x_{1} \in V_{1}$ and $x_{0} \in \mathcal{X}(T)$. Since $\|\cdot\|^{\prime}$ is equivalent to the original norm, there is a positive real number $\alpha$, such that $\alpha\|x\|^{\prime} \leqslant\|x\|$ or $\alpha\left[\left\|x_{1}\right\|+\left\|x_{0}\right\|\right] \leqslant\|x\|$.

Because the sequence $\left\{\left\|\tilde{M}_{n}^{-1}\right\|\right\}$ is bounded, there is a positive number $\lambda$, such that $\left\|\tilde{M}_{n}^{-1}\right\|^{-1} \geqslant \lambda$. Hence, $\lambda\|x\| \leqslant\left\|\tilde{M}_{n}^{-1}\right\|^{-1}\|x\| \leqslant\left\|\tilde{M}_{n}(x)\right\|$ for all $x \in V$ and for all $\tilde{M}_{n}$. Let $\varepsilon$ and $\delta$ denote positive real numbers such that $2 \delta<\varepsilon<\alpha \lambda / 3$ and let $M$ be a member of the given sequence $\left\{\tilde{M}_{n}\right\}$ such that 
$\left\|\left.M\right|_{V_{1}}-\left.T\right|_{V_{1}}\right\|<\varepsilon$. Denote by $N_{1}$ the subset of $\mathscr{R}(T)$ which contains all elements of $\mathcal{T}(T)$ with norm one.

It will now be shown that:

$$
\begin{aligned}
& \text { if } x_{0} \in N_{1} \text {, then there is a } y \in V \text {, such that } \\
& \left\|y-M\left(x_{0}\right)\right\|<\varepsilon \text { and }\|y-z\| \geqslant \delta \text { for all } z \in R(T) .
\end{aligned}
$$

Case 1. $M\left(x_{0}\right) \notin \Re(T)$. Now, $\rho=\inf \left\{\left\|M\left(x_{0}\right)-z\right\|: z \in \Re(T)\right\}>0$. If $\rho \geqslant \delta$, let $y=M\left(x_{0}\right)$. If $\rho<\delta$, then by [10, Lemma 1.7, p. 86], there is $u \in V$ such that $\|u\|=1$ and $\|u-z\|>\beta$, for all $z \in R(T)$, where $2 \delta / \varepsilon<\beta<1$. Let $\gamma$ denote a real number such that $1-\varepsilon<\gamma<1-2 \delta / \beta$. Then let $y=(1-\gamma) u+M\left(x_{0}\right)$.

Case 2. $M\left(x_{0}\right) \in \mathcal{R}(T)$. Let $\beta$ and $\gamma$ denote real numbers such that $\delta / \varepsilon<\beta<1$ and $1-\varepsilon<\gamma<1-\delta / \beta$. Let $u$ be defined as in Case 1 and, again, let $y=(1-\gamma) u+M\left(x_{0}\right)$. Then it follows that $(*)$ is true.

Let $\mathcal{S}$ denote the set of one-to-one, bounded, linear, extensions, $S$, of $T_{1}=\left.T\right|_{\nu_{1}}$ such that if $x \in N_{1} \cap \operatorname{dom}(S)$ and $z \in \Re(T)$, then $\| S(x)-$ $M(x) \| \leqslant \varepsilon$ and $\|S(x)-z\| \geqslant \delta$. It will now be shown that $\delta \neq \varnothing$. Let $x_{0} \in N_{1}$ and let $y$, which depends on $x_{0}$, be as in (*). Denote by $\left\langle x_{0}\right\rangle$ the subspace of $V$ generated by $x_{0}$. Define $S: V_{1} \oplus\left\langle x_{0}\right\rangle \rightarrow \Re(T) \oplus\langle y\rangle$ by $S\left(x_{1}+\omega x_{0}\right)=T_{1}\left(x_{1}\right)+\omega y$, where $x_{1} \in V_{1}$ and $\omega$ is a complex number. Then $S \in \mathcal{S}$.

Partially order $\mathcal{S}$ by $S_{1} \leqslant S_{2}$ if and only if $\operatorname{dom}\left(S_{1}\right) \subseteq \operatorname{dom}\left(S_{2}\right)$ and $S_{2}$ is an extension of $S_{1}$. Let $\mathcal{C}$ be a chain in $\mathcal{S}$ and let $D_{0}=\cup_{S \in \mathcal{C}} \operatorname{dom}(S)$. Define $S_{0}: D_{0} \rightarrow V$ by $S_{0}(x)=S(x)$ if $x \in \operatorname{dom}(S)$ and $S \in \mathcal{C}$. To see that $S_{0}$ is well defined, suppose that $x \in \operatorname{dom}\left(S_{1}\right) \cap \operatorname{dom}\left(S_{2}\right)$ for some $S_{1}, S_{2} \in$ $C$. Since $C$ is a chain, it may be assumed without loss of generality that $S_{1} \leqslant S_{2}$. Hence, $x \in \operatorname{dom}\left(S_{1}\right) \cap \operatorname{dom}\left(S_{2}\right)=\operatorname{dom}\left(S_{1}\right)$, and so $S_{1}(x)=S_{2}(x)$ $=S_{0}(x)$, which implies that $S_{0}$ is well defined. If $x, y \in D_{0}$, then $x, y \in$ $\operatorname{dom}(S)$ for some $S \in \mathcal{S}$. For any complex number $\omega$,

$$
S_{0}(\omega x+y)=S(\omega x+y)=\omega S(x)+S(y)=\omega S_{0}(x)+S_{0}(y) .
$$

Therefore, $S_{0}$ is linear. If $S_{0}(x)=\theta$, then $S(x)=\theta$, which implies that $x=\theta$. Thus, $S_{0}$ is one-to-one on $D_{0}$. Since each $S$ in $C$ is an extension of $T_{1}, S_{0}$ is also an extension of $T_{1}$ in addition to being an extension of each $S$ in $C$. If $x_{0} \in N_{1} \cap \operatorname{dom}\left(S_{0}\right)$, then $x_{0} \in N_{1} \cap \operatorname{dom}(S)$ for some $S \in \mathcal{C}$. Hence, $\left\|S_{0}\left(x_{0}\right)-M\left(x_{0}\right)\right\|=\left\|S\left(x_{0}\right)-M\left(x_{0}\right)\right\| \leqslant \varepsilon$, and for $z \in \mathcal{R}(T), \| S_{0}\left(x_{0}\right)-$ $z\|=\| S\left(x_{0}\right)-z \| \geqslant \delta$. If $x=x_{1}+x_{0} \in \operatorname{dom}\left(S_{0}\right)$, where $x_{1} \in V_{1}$ and $x_{0} \in$ $\mathfrak{R}(T)$, then $x, x_{1}, x_{0} \in \operatorname{dom}(S)$ for some $S \in \mathcal{C}$. Hence,

$$
\begin{aligned}
\left\|M(x)-S_{0}(x)\right\| & \leqslant\left\|M\left(x_{1}\right)-S_{0}\left(x_{1}\right)\right\|+\left\|M\left(x_{0}\right)-S_{0}\left(x_{0}\right)\right\| \\
& \leqslant\left\|\left.M\right|_{V_{1}}-\left.T\right|_{V_{1}}\right\|\left\|x_{1}\right\|+\varepsilon\left\|x_{0}\right\| \\
& \leqslant \varepsilon\left\|x_{1}\right\|+\varepsilon\left\|x_{0}\right\|=\varepsilon\left[\left\|x_{1}\right\|+\left\|x_{0}\right\|\right] \leqslant\|x\| \varepsilon / \alpha .
\end{aligned}
$$

Thus, 


$$
\left\|S_{0}(x)\right\| \leqslant\|M(x)\|+\|x\| \varepsilon / \alpha \leqslant[\|M\|+\varepsilon / \alpha]\|x\|,
$$

which implies that $S_{0}$ is bounded. Therefore, $S_{0} \in \mathcal{S}$, and since $S \leqslant S_{0}$, for all $S \in \mathcal{C}$, the chain $\mathcal{C}$ has an upper bound. Then, by Zorn's Lemma, $\delta$ has a maximal element, say $S^{\prime}$. Let $D^{\prime}$ denote the domain of $S^{\prime}$.

Now it will be proven that:

$$
\text { there is a positive number } \xi \text {, such that }
$$

$$
\xi\|x\| \leqslant\left\|S^{\prime}(x)\right\| \text {, for all } x \in D^{\prime} .
$$

If not, then there is a sequence $\left\{y_{n}\right\}$ in $D^{\prime}$ such that $\left\|y_{n}\right\|=1$ and $\left\|S^{\prime}\left(y_{n}\right)\right\|$ $<1 / n$. Thus, $\lim _{n}\left[S^{\prime}\left(y_{n}\right)\right]=\theta$, where $\theta$ denotes the zero element of $V$. Let $y_{n}=y_{n 1}+y_{n 0}$, where $y_{n 1} \in V_{1}$ and $y_{n 0} \in \Re(T)$. For $y_{n 0} \neq \theta$,

$$
\begin{aligned}
1 / n> & >S^{\prime}\left(y_{n}\right)\|=\| y_{n 0}\|\| S^{\prime}\left(y_{n 0} /\left\|y_{n 0}\right\|\right)+S^{\prime}\left(y_{n 1} /\left\|y_{n 0}\right\|\right) \| \\
& =\left\|y_{n 0}\right\|\left\|S^{\prime}\left(y_{n 0} /\left\|y_{n 0}\right\|\right)-T\left(-y_{n 1} /\left\|y_{n 0}\right\|\right)\right\| \geqslant\left\|y_{n 0}\right\| \delta,
\end{aligned}
$$

since $y_{n 0} /\left\|y_{n 0}\right\| \in N_{1} \cap \operatorname{dom}\left(S^{\prime}\right)$ and $T\left(-y_{n 1} /\left\|y_{n 0}\right\|\right) \in \mathscr{R}(T)$. Hence, $\lim _{n}\left[y_{n 0}\right]=\theta$. $S^{\prime}$ bounded implies that $\lim _{n}\left[S^{\prime}\left(y_{n 0}\right)\right]=\theta$. This, along with the fact that $\lim _{n}\left[S^{\prime}\left(y_{n}\right)\right]=\theta$ implies that $\lim _{n}\left[T_{1}\left(y_{n 1}\right)\right]=\lim _{n}\left[S^{\prime}\left(y_{n 1}\right)\right]=\theta$. Since $T_{1}$ is a homeomorphism defined on $V_{1}, \lim _{n}\left[y_{n 1}\right]=\theta$. Thus, $\lim _{n}\left[y_{n}\right]=$ $\theta$, which contradicts the fact that $\left\|y_{n}\right\|=1$. Hence, $(* *)$ is true. Now, $S^{\prime}$ can be extended to the closure of $D^{\prime}$ be defining $S^{\prime \prime}: \operatorname{cl}\left(D^{\prime}\right) \rightarrow V$ by $S^{\prime \prime}(x)=$ $\lim _{n}\left[S^{\prime}\left(x_{n}\right)\right]$, where $x_{n} \in D^{\prime}$ and $\lim _{n}\left[x_{n}\right]=x . S^{\prime \prime}$ is a bounded linear extension of both $T_{1}$ and $S^{\prime}$, with $\left\|S^{\prime}\right\|=\left\|S^{\prime \prime}\right\|$, [2, p. 279]. If $x \in \operatorname{cl}\left(D^{\prime}\right)$ and $x_{n} \in D^{\prime}$ with $\lim _{n}\left[x_{n}\right]=x$, then $\lim _{n}\left[S^{\prime}\left(x_{n}\right)\right]=S^{\prime \prime}(x)$ and $\xi\left\|x_{n}\right\| \leqslant\left\|S^{\prime}\left(x_{n}\right)\right\|$. Thus, $\xi\|x\| \leqslant\left\|S^{\prime \prime}(x)\right\|$. By [11, Theorem 3.1-B, p. 86], the inverse of $S^{\prime \prime}$ exists and is bounded on its domain, $\Re\left(S^{\prime \prime}\right)$. It follows that $S^{\prime \prime} \in \mathcal{S}$. Since $S^{\prime} \leqslant S^{\prime \prime}$ and $S^{\prime}$ is a maximal element of $\mathcal{S}$, then $S^{\prime}=S^{\prime \prime}$ and $D^{\prime}$ is closed. The inverse of $S^{\prime}$ being bounded implies $\Re\left(S^{\prime}\right)$ is closed. If $x=x_{1}+x_{0} \in$ $D^{\prime}$, where $x_{1} \in V_{1}$ and $x_{0} \in \Re(T)$, then

$$
\begin{aligned}
\left\|M(x)-S^{\prime}(x)\right\| & \leqslant\left\|M\left(x_{1}\right)-T_{1}\left(x_{1}\right)\right\|+\left\|M\left(x_{0}\right)-S^{\prime}\left(x_{0}\right)\right\| \\
& <\varepsilon\left[\left\|x_{1}\right\|+\left\|x_{0}\right\|\right] \leqslant\|x\| \varepsilon / \alpha,
\end{aligned}
$$

which compares $\mathcal{R}\left(S^{\prime}\right)$ and $M\left(D^{\prime}\right)$ and will be used later.

Consider the following possibilities:

(a) $D^{\prime}$ and $R\left(S^{\prime}\right)$ are proper closed subspaces of $V$,

(b) $D^{\prime}=V$ and $R\left(S^{\prime}\right) \neq V$,

(c) $D^{\prime} \neq V$ and $\Re\left(S^{\prime}\right)=V$,

(d) $D^{\prime}=V$ and $R\left(S^{\prime}\right)=V$.

If $D^{\prime}$ and $\Re\left(S^{\prime}\right)$ are proper subspaces of $V$, then $S^{\prime}$ can be extended as $T_{1}$ was extended in the proof that $\delta \neq \varnothing$, which contradicts the maximality of $S^{\prime}$.

If $D^{\prime}=V$ and $\Re\left(S^{\prime}\right) \neq V$, then since $\mathcal{R}\left(S^{\prime}\right)$ is closed, by [10, p. 86], there is $y \in V$ such that $\|y\|=1$ and $\|y-z\|>\beta$, for all $z \in \mathcal{R}\left(S^{\prime}\right)$, where $\varepsilon /(\alpha \lambda-2 \varepsilon)<\beta<1$. Let $x=M^{-1}(y)$. Then $\beta<\left\|M(x)-S^{\prime}(x)\right\| \leqslant$ $\|x\| \varepsilon / \alpha$. Hence, $\|x\|>\alpha \beta / \varepsilon$. $\left\|M(x)-S^{\prime}(x)\right\|<\|x\| \varepsilon / \alpha$ and $\lambda\|x\| \leqslant$ 
$\|M(x)\|$ imply that $\left\|S^{\prime}(x)\right\| \geqslant\|x\|(\lambda-\varepsilon / \alpha)$. Hence

$$
\|x\|(\lambda-\varepsilon / \alpha) \leqslant\left\|M(x)-S^{\prime}(x)\right\|+\|M(x)\| \leqslant\|x\| \varepsilon / \alpha+1 .
$$

Therefore, $\lambda-\varepsilon / \alpha \leqslant \varepsilon / \alpha+\|x\|^{-1}<\varepsilon / \alpha+\varepsilon / \alpha \beta$ or $\beta<\varepsilon /(\alpha \lambda-2 \varepsilon)$, which contradicts the choice of $\beta$, namely, $\varepsilon /(\alpha \lambda-2 \varepsilon)<\beta$.

If $D^{\prime} \neq V$ and $\Re\left(S^{\prime}\right)=V$, let $\beta^{\prime}$ denote a real number such that $\varepsilon /(\alpha \lambda-$ $\varepsilon)<\beta^{\prime}<1$. Since $D^{\prime}$ is closed, $M\left(D^{\prime}\right)$ is also closed. Hence, there is $y^{\prime} \in V$ such that $\left\|y^{\prime}\right\|=1$ and $\left\|y^{\prime}-z\right\|>\beta^{\prime}$ for all $z \in M\left(D^{\prime}\right)$. Let $x^{\prime}=S^{\prime-1}\left(y^{\prime}\right)$. Then $\beta^{\prime}<\left\|M\left(x^{\prime}\right)-S^{\prime}\left(x^{\prime}\right)\right\| \leqslant\left\|x^{\prime}\right\| \varepsilon / \alpha$ or $\left\|x^{\prime}\right\|>\alpha \beta^{\prime} / \varepsilon$. Now,

$$
\lambda\left\|x^{\prime}\right\|<\left\|M\left(x^{\prime}\right)\right\| \leqslant\left\|M\left(x^{\prime}\right)-S^{\prime}\left(x^{\prime}\right)\right\|+\left\|S^{\prime}\left(x^{\prime}\right)\right\| \leqslant\left\|x^{\prime}\right\| \varepsilon / \alpha+1 .
$$

Hence, $\lambda \leqslant \varepsilon / \alpha+\left\|x^{\prime}\right\|^{-1}<\varepsilon / \alpha+\varepsilon / \alpha \beta^{\prime}$ or $\beta^{\prime}<\varepsilon /(\alpha \lambda-\varepsilon)$, which contradicts the choice of $\beta^{\prime}$.

Therefore $D^{\prime}=V$ and $\mathscr{R}\left(S^{\prime}\right)=V$ is the only possibility and $S^{\prime} \in \mathfrak{N}$.

((ii) implies (iii).) Let $P$ denote the projection of $V$ onto $V_{1}$ along $\mathscr{T}(T)$ and let $I$ denote the identity operator on $V$. Then $S_{n}=S^{\prime} P+n^{-1} S^{\prime}(I-P)$ $\in \mathscr{T}$ and $\lim _{n}\left[S_{n}\right]=T$. Therefore, $T \in \mathscr{B}$.

((iii) implies (i).) Define another norm $\|\cdot\|^{\prime \prime}$ on $V$ by $\|x\|^{\prime \prime}=\max \left\{\left\|x_{1}\right\|\right.$, $\left.\left\|x_{0}\right\|\right\}$, where $x=x_{1}+x_{0}$ with $x_{1} \in V_{1}$ and $x_{0} \in \Re(T)$. Let $V^{\prime \prime}$ denote $V$ with norm $\|\cdot\|^{\prime \prime}$. Then the function $\phi$, defined from $V^{\prime \prime}$ onto $V$ by $\phi(x)=x$, is a linear homeomorphism. The three spaces, $B[V], B\left[V^{\prime \prime}, V\right]$ and $B\left[V, V^{\prime \prime}\right]$ are linearly homeomorphic with $M \in B[V]$ corresponding to $M \phi \in$ $B\left[V^{\prime \prime}, V\right]$ and $\phi^{-1} M \in B\left[V, V^{\prime \prime}\right]$. Hence,

$$
\begin{aligned}
& \|M \phi\|=\sup \left\{\|M(x)\|:\|x\|^{\prime \prime}=1\right\}, \quad \text { and } \\
& \left\|(M \phi)^{-1}\right\|^{-1}=\inf \left\{\|M(x)\|:\|x\|^{\prime \prime}=1\right\} .
\end{aligned}
$$

For $M \in \mathfrak{N}$, let $m_{1}=\inf \left\{\left\|M\left(x_{1}\right)\right\|: x_{1} \in V_{1}\right.$ and $\left.\left\|x_{1}\right\|=1\right\}$ and $m_{0}=$ $\inf \left\{\left\|M\left(x_{0}\right)\right\|: x_{0} \in \mathcal{R}(T)\right.$ and $\left.\left\|x_{0}\right\|=1\right\}$. Define $\tilde{M} \in \Re$ by $\tilde{M}=M P+$ $\left(m_{1} / m_{0}\right) M(I-P)$. Then,

$$
\begin{aligned}
\left\|(\tilde{M} \phi)^{-1}\right\|^{-1} & =\inf \left\{\|\tilde{M}(x)\|:\|x\|^{\prime \prime}=1\right\} \\
& =\inf \left\{\left\|M\left(x_{1}\right)\right\|: x_{1} \in V_{1} \text { and }\left\|x_{1}\right\|=1\right\} .
\end{aligned}
$$

Since $T_{1}=\left.T\right|_{V_{1}}$ is a linear homeomorphism from $V_{1}$ onto $R(T), T_{1}^{-1}$ exists. $T \in \mathscr{B}$ implies that there is a sequence $\left\{M_{n}\right\}$ in $\mathscr{N}$ such that $\lim _{n}\left[M_{n}\right]=T$. Let $\varepsilon$ denote a positive real number less than $\left\|T_{1}^{-1}\right\|^{-1}$. Then there is a positive real number $N$, such that if $n \geqslant N$, then $\left\|T(x)-M_{n}(x)\right\|$ $<\varepsilon\|x\|$ for all $x \in V$. Then

$$
\left\|T_{1}^{-1}\right\|^{-1}-\varepsilon \leqslant\left\|\left(\tilde{M}_{n} \phi\right)^{-1}\right\|^{-1}=\left\|\phi^{-1} \tilde{M}_{n}^{-1}\right\|^{-1} \text { for } n \geqslant N .
$$

Thus, $\left\{\phi^{-1} \tilde{M}_{n}^{-1}\right\}$ is a sequence in $B\left[V, V^{\prime \prime}\right]$ such that $\left\{\left\|\phi^{-1} \tilde{M}_{n}^{-1}\right\|\right\}$ is bounded and, hence, the corresponding sequence in $B[V]$, namely $\left\{\tilde{M}_{n}^{-1}\right\}$, is such that $\left\{\left\|\tilde{M}_{n}^{-1}\right\|\right\}$ is bounded. Since $\left.\tilde{M}_{n}\right|_{V_{1}}=\left.M_{n}\right|_{V_{1}}, \lim _{n}\left[\left.\tilde{M}_{n}\right|_{V_{1}}\right]=T_{1}$.

((ii) implies (iv).) $S^{\prime} \in \mathscr{N}$ implies that $V=S^{\prime}\left(V_{1}\right) \oplus S^{\prime}(\Re(T))=\mathscr{R}(T)$ 
$\bigoplus S^{\prime}(\mathscr{R}(T))$. Thus, $T \in G F[V]$ and $S^{\prime}(\Re(T))$ is a closed complement of $\Re(T)$ that is homeomorphic to $\Re(T)$.

((iv) implies (iii).) This is Corollary 2.2 of [6]. Thus, the proof of the theorem is complete.

Let $\mathcal{P}$ denote the set of bounded projections on $V$, other than the identity operator and the null operator, let $\mathscr{N} \mathscr{P}=\{M P: M \in \mathscr{N}$ and $P \in \mathscr{P}\}$ and let $\mathscr{P} \mathfrak{N}=\{P M: P \in \mathscr{P}$ and $M \in \mathscr{N}\}$.

Corollary. $\mathscr{B} \cap G[V]=\mathscr{T} \mathscr{P}=\mathscr{P} \mathfrak{T}$.

Proof. This follows from the observation that for $T$ as in the hypothesis of the theorem, (ii) can be stated as:

(ii)' there is $S \in \mathfrak{T}$ such that $T=S P$, where $P$ is the projection of $V$ onto $V_{1}$ along $\Re(T)$.

3. Comments. The equivalence of (ii)' and (iv) is a special case of Theorem 1 of [3]. In [3], it is noted that in order to extend $T_{1}$, a linear homeomorphism between $\mathscr{R}(T)$ and a closed complement of $\Re(T)$ is needed. If the above theorem is viewed as a theorem about generalized Fredholm operators, then the fact that the operator is also in $\mathcal{B}$ is sufficient to produce this homeomorphism even though it is only assumed that the operator is in $G[V]$. If the above theorem is viewed as a theorem about operators in $\mathscr{B}$, it provides the fact that $\mathscr{B} \cap G[V]$ is the same as $\{T \in G F[V]: \mathcal{N}(T)$ is linearly homeomorphic to a closed complement of $\Re(T)\}$.

As noted in [4], the hypothesis that $T \in G[V]$ is satisfied if $V$ is a Hilbert space and if $\mathscr{R}(T)$ is closed. The sufficient condition for $T \in \mathscr{B}$ given in [7] is $V=\mathscr{R}(T) \oplus \mathfrak{R}(T)$, which is a special case for $T \in G F[V]$.

\section{REFERENCES}

1. F. V. Atkinson, On relatively regular operators, Acta Sci. Math. (Szeged) 15 (1953), 38-56.

2. G. Bachman and L. Narici, Fundamental analysis, Academic Press, New York, 1966.

3. F. J. Beutler, The operator theory of the pseudo-inverse, J. Math. Anal. Appl. 10 (1965), 457-470, 471-493.

4. S. R. Caradus, Perturbation theory for generalized Fredholm operators, Pacific J. Math. 52 (1974), 11-15.

5. J. Feldman and R. V. Kadison, The closure of the regular operators in a ring of operators, Proc. Amer. Math. Soc. 5 (1954), 909-916.

6. D. A. Hogan and C. E. Langenhop, Invertibility in a Banach algebra, Indiana Univ. Math. J. 24 (1975), 965-977.

7. E. P. Kelly, Jr. and D. A. Hogan, Bounded, conservative, linear operators and the maximal group. II, Proc. Amer. Math. Soc. 38 (1973), 298-302.

8. M. Z. Nashed and G. F. Votruba, $A$ united approach to generalized inverses of linear operators, Bull. Amer. Math. Soc. 80 (1974), 825-830, 831-835.

9. B. E. Rhoades, Triangular summability methods and the boundary of the maximal group, Math. Z. 105 (1968), 284-290.

10. M. Schechter, Principles of functional analysis, Academic Press, New York, 1971.

11. A. E. Taylor, Introduction to functional analysis, Wiley, New York, 1958.

Department of Mathematics, Louisiana Tech University, Ruston, Louisiana 71272 